\title{
Predictores de síntomas del TEPT en cuidadores de pacientes pediátricos sobrevivientes de quemaduras
}

\section{Predictors of PTSD symptoms in caregivers of pediatric burn survivors}

\author{
Lucía Quezada Berumen $^{1}$ \\ Mónica González Ramírez \\ Gabriel Mecott Rivera \\ Universidad Autónoma de Nuevo León, México \\ (Rec: septiembre de 2015 - Acept: noviembre de 2015)
}

\begin{abstract}
Resumen
Enfrentarse a una lesión grave en los hijos resulta una de las experiencias más devastadoras que los padres y madres pueden enfrentar. El presente estudio exploró el rol de la resiliencia en padres y madres de niños con quemaduras, el TBSA quemado, la edad al momento de la quemadura y el tiempo desde la quemadura, sobre la sintomatología del trastorno de estrés postraumático (TEPT) de los cuidadores. Se trata de un estudio transversal donde un total de 51 padres, madres y tutoras de pacientes con quemaduras fueron evaluados. Los resultados mostraron que a mayor fortaleza y confianza en los cuidadores, menor severidad en su sintomatología del TEPT. Las reacciones post-quemadura de los padres y cuidadores pueden repercutir en el bienestar de sus hijos, por lo que a mayor conocimiento sobre los factores relacionados con la adaptación de estos cuidadores, mejor atención por parte de los servicios de salud.
\end{abstract}

Palabras clave: síntomas del TEPT, quemaduras, cuidadores, resiliencia.

\begin{abstract}
Facing a severe injury in the children is one of the most devastating experiences that parents may face. The aim of this study was to explore the role of resilience showed by fathers and mothers of children with burns, the TBSA burned, age at the time of the burn and time since the burn in PTSD symptoms in caregivers. It was a cross-sectional study where fathers, mothers and guardians of 51 burn patients were evaluated. Results showed that the higher strength and confidence in caregivers, less severity in PTSD symptoms. The post-burn reactions of parents and guardians can affect the responses and welfare of their children. Therefore, a better understanding of factors related to the adaptation in caregivers, better attention by health services.
\end{abstract}

Keywords: PTSD symptoms, burns, caregivers, resilience.

\footnotetext{
1 Correspondencia a: Lucía Quezada Berumen. Universidad Autónoma de Nuevo León (UANL), Facultad de Psicología. Avenida Universidad (sin número), Ciudad Universitaria San Nicolás de Garza C.P. 66451, Nuevo León, Mexico. E-mail: luciaqb86@ msn.com.
} 


\section{Introducción}

En México las quemaduras se sitúan dentro de las 20 principales causas de enfermedades no transmisibles en la población general, ocupando el séptimo lugar. En el 2011 se registraron un total de 129.779 casos de accidentes por quemaduras, los cuales afectaron ligeramente más a los hombres que a las mujeres. El estado de Nuevo León, ocupa el primer lugar en las tasas de incidencia respecto al promedio nacional (118.82), con una incidencia de 205.51 por cada 100000 habitantes, seguido por Sonora (201.34) y Coahuila (192.27) (Dirección General de Epidemiología, 2012).

Las quemaduras se han descrito como una de las lesiones más serias y traumáticas que los niños y adolescentes pueden experimentar (Saxe et al., 2005), las cuales afectan tanto a su bienestar como al de sus familias (Bakker, Maertens, Van Son \& Van Loey, 2013). Un hallazgo importante en el campo de las lesiones pediátricas no intencionales, es que tanto madres y padres se ven igualmente o incluso más afectados que el niño (Kazak et al., 2006; Langeland \& Olff, 2008). Ser testigo del evento de la quemadura, el temor por la vida del niño, presenciar el dolor de éste y los graves daños en la piel, así como experimentar sentimientos de incertidumbre y falta de control sobre el futuro puede resultar una experiencia abrumadora para ambos padres. De esta manera, enfrentar un evento que involucra lesiones graves en un niño se especifica como un evento traumático para padres y madres (American Psychiatric Association, 2000).

En estudios realizados mayoritariamente en madres, se han encontrado reacciones emocionales y psicológicas adversas, después del accidente de la quemadura de sus hijos, como síntomas de estrés agudo, ira, sentimientos de culpa, depresión, ansiedad y trastorno de estrés postraumático (TEPT en adelante; Bakker, Van Loey, Van der Heijden, \& Van Son, 2012; Bakker, Van Loey, Van Son, \& Van der Heijden, 2010; El Hamaoui, Yaalaoui, Chihabeddine, Boukind \& Moussaoui, 2006; Phillips \& Rumsey, 2008; Rizzone, Stoddard, Murphy \& Kruger, 1994).

Se ha sugerido que todos los padres y madres corren el riesgo de desarrollar efectos psicológicos adversos, independientemente del tamaño o severidad de la quemadura de su hijo (Phillips \& Rumsey, 2008). Rizzone et al. (1994) encontraron que el principal predictor de la sintomatología del TEPT en madres y padres de estos niños fue el total de superficie corporal afectada (TBSA en adelante). Asimismo, reportaron que un $72 \%$ de las madres y padres experimentaron dichos síntomas dentro de los 6 meses posteriores a la quemadura de sus hijos, donde un 56\% continuó experimentándolos años después del accidente. Los síntomas del TEPT tanto en madres como en padres de estos pacientes se han relacionado con la edad del niño al momento de la quemadura. De este modo, una mayor sintomatología parental se asoció con una menor edad en los niños al momento de la lesión (Odar et al., 2013).

Las lesiones por quemaduras producen una amplia variedad de desafíos tanto para los niños y adolescentes como para sus padres y madres. Se ha propuesto que la presencia y manifestación de reacciones negativas en el paciente pediátrico se ven ciertamente influidas por la forma en que los padres y madres se adapten a la situación de la quemadura. Esta influencia parental, generalmente, se debe a que en los niños pequeños resulta de capital importancia la valoración de la situación que hagan sus padres o cuidadores, pues una respuesta positiva de estos puede amortiguar el impacto estresante sobre el niño y viceversa (Breton, Valla \& Lambert, 1993). De acuerdo a Olsson, Bond, Burns, Vella-Brodrick y Sawyer (2003), la resiliencia se piensa como un factor protector que puede amortiguar las consecuencias psicológicas negativas como el TEPT.

Las consecuencias psicológicas adversas en padres y madres de niños sobrevivientes de quemaduras se encuentran bien documentadas. Aunque el TBSA se ha reportado como un predictor de la sintomatología del TEPT, hasta donde nuestro conocimiento llega, otras variables predictoras aún no han sido objeto de estudio. Así, con la identificación de estas variables en los padres y madres, se obtendría información importante para el desarrollo de acciones preventivas y de intervención, las cuales permitan el manejo de estos síntomas de manera eficaz y logren repercutir de manera positiva sobre sus hijos.

Por lo anterior, el propósito de este estudio consistió en determinar los predictores de la sintomatología del TEPT en los padres y madres de niños y adolescentes sobrevivientes de quemaduras, utilizando: 1) el total de superficie corporal afectada (TBSA) de los hijos como variable médica, 2) el tiempo transcurrido desde la quemadura como variable del evento, 3) la edad al momento de la quemadura como variable de los hijos y 4) la resiliencia parental como variable psicológica. 


\section{Método}

\section{Procedimiento}

Este trabajo consistió en un estudio transversal donde participaron díadas de pacientes pediátricos sobrevivientes de quemaduras y sus respectivos padres, madres o tutores. Los criterios de inclusión para los pacientes fueron que tuvieran una edad de 7 a 18 años, un periodo de tiempo mayor a los 3 meses desde el evento de la quemadura y que asistieran al Servicio de Cirugía Plástica Reconstructiva y Maxilofacial del Hospital de la Universidad Autónoma de Nuevo León (UANL). Para el grupo de padres, madres y tutores, se requirió que contaran con habilidades de lecto-escritura para dar respuesta a los instrumentos de evaluación.

La aprobación del Comité de Ética y de Investigación de la Facultad de Medicina y el Hospital Universitario de la UANL fue obtenida. La recolección de los datos fue llevada a cabo en el 2013 durante las citas de las clínicas ambulatorias de atención a secuelas por quemaduras para pacientes pediátricos, realizadas dentro del hospital. Durante el tiempo de espera previo a la evaluación médica, aquellos participantes que cumplieran con los criterios de inclusión, fueron invitados a participar de manera voluntaria y sin ninguna retribución económica. Para el propósito del estudio, díadas entre "paciente-padre, madre o tutor" fueron requeridas. Si ambos padres o tutores estuvieron presentes, se solicitó la participación de uno sólo para dar respuesta a los instrumentos de evaluación del estudio; el investigador sugirió que aquel que pasara más tiempo con el paciente fuera quien completara los cuestionarios (de aquí en adelante denominado como "cuidador").

\section{Participantes}

Se contó con la participación de 51 díadas "paciente-cuidador". Los pacientes tuvieron una media de edad de 12.24 años al momento de la evaluación, un promedio de 6.4 años transcurridos desde el evento de la quemadura y una media de 5.8 años al momento de la lesión. El TBSA quemado fue de $31 \%$ en promedio. Participaron 29 hombres y 22 mujeres con un promedio de edad al momento de la evaluación de 12 y 13 años respectivamente.

Los cuidadores tuvieron una edad promedio de 38.96 años al momento de la evaluación. La muestra incluyó a 39 mujeres, con una media de 36.85 años y estuvo compuesta por 35 madres de familia, tres abuelas y una hermana. Doce padres de familia participaron, con una promedio de edad de 45.83 años. De acuerdo al estado civil, el $49 \%$ se encontraba casado(a), el $19.6 \%$ en unión libre, el $7.8 \%$ divorciado (a), el $11.8 \%$ separado(a), un $7.9 \%$ eran mujeres solteras y el $3.9 \%$ viudas.

\section{Instrumentos}

La Escala de Resiliencia Mexicana para adultos (RESI-M) de Palomar y Gómez (2010) es una escala de auto-reporte que evalúa la capacidad de lidiar con el estrés y la adversidad. Este instrumento consiste en 43 ítems con opciones de respuesta tipo Likert, con puntajes que van desde 43 hasta 172; los puntajes más elevados indican un mayor nivel de resiliencia. La escala se divide en cinco factores: Fortaleza y confianza en sí mismo, Competencia social, Apoyo familiar, Apoyo social y Estructura, donde su consistencia interna oscila de $\alpha=.79$ a $\alpha=.92$. La consistencia interna para la escala completa es alta $(\alpha=.93)$.

La Escala de Trauma de Davidson (DTS), de Davidson et al. (1997), en su versión española validada por Bobes et al. (2000), está compuesta por 17 ítems que corresponden y evalúan cada uno de los 17 síntomas pertenecientes a los criterios B, C y D contenidos en el DSM-IV para el diagnóstico del TEPT. Esta es una escala de auto-reporte que evalúa la severidad de la sintomatología del TEPT en la cual la persona debe puntuar dos aspectos en cada ítem (frecuencia y gravedad). El marco de referencia temporal que los sujetos deben considerar es la semana previa. El rango de respuestas para el total de la escala es de 0 a 136 puntos. Los autores de la DTS proponen como punto de corte más eficiente los 40 puntos, los cuales muestran una sensibilidad del $69 \%$, una especificidad del $95 \%$, un valor predictivo positivo del $92 \%$ y un valor predictivo negativo del $83 \%$. El coeficiente alpha de Cronbach es de .89 para el total del instrumento.

\section{Datos sobre la lesión por quemadura}

Información sobre la lesión por quemadura se obtuvo de los registros médicos de los pacientes, incluyendo el TBSA quemado y la fecha de la lesión.

\section{Análisis de los datos}

Los estadísticos de la prueba de KolmogorovSmirnov, indicaron que ninguna de las variables se distribuyó normalmente, por lo que los datos se describen 
con estadísticos no paramétricos. El coeficiente de correlación de Spearman se utilizó para estimar la relaciones entre las variables, con un valor de $\mathrm{p}<.05$ para ser consideradas estadísticamente significativas. Los análisis de correlación así como los análisis descriptivos se realizaron en SPSS 20. Para identificar los predictores de la sintomatología del TEPT, se trabajó con modelamiento de ecuaciones estructurales (SEM) utilizando SPSS Amos 18. Para que los parámetros dentro del modelo fueran considerados estadísticamente significativos, se consideró un valor de $\mathrm{p}<.05$. Para probar el ajuste de los modelos, se siguieron los límites recomendados por Hooper, Coughlan y Mullen (2008) con los siguientes índices de ajuste: para el cociente entre ji-cuadrado y sus grados de libertad $(\chi 2 / g l)$, valores $<3$; para los índices de bondad de ajuste (GFI) de Jöreskog y Sörbom y su modalidad corregida (AGFI), índice de ajuste normativo (NFI), índice de Tucker-Lewis (TLI) y el índice de ajuste comparativo (CFI), con valores $\geq .95$. Finalmente, para el residuo cuadrático medio de aproximación (RMSEA) de Steiger-Lind, valores < 07 .

\section{Resultados}

Severidad de la sintomatología del TEPT en los cuidadores

Los datos presentados en la Tabla 1 muestran que los cuidadores obtuvieron una media en su puntaje por debajo del punto de corte propuesto por los autores de la DTS para la detección de posibles casos clínicos. Por parentesco con el paciente, las tutoras fueron quienes presentaron mayor severidad en los tres grupos de síntomas, seguidas de las madres y finalmente los padres.

Tabla 1

Puntaje en la escala total y por grupos de síntomas del TEPT

\begin{tabular}{lccc}
\hline & Mínimo & Máximo & M \\
\hline Escala Total & 1 & 83 & 32.03 \\
Reexperimentación & 0 & 28 & 10.82 \\
Evitación & 0 & 28 & 10.55 \\
Activación & 0 & 32 & 10.67 \\
\hline
\end{tabular}

\section{Niveles de resiliencia de los cuidadores}

En la Tabla 2 se presentan los puntajes obtenidos por los cuidadores en la RESI-M tanto para el total de la escala como para los cinco factores que la conforman. Cabe destacar que la RESI-M no cuenta con puntos de corte establecidos, por lo que a altos puntajes en la escala corresponde una mayor resiliencia. Tomando en cuenta que la puntuación máxima posible de la RESI-M es de 172, se concluyó que los cuidadores presentaron un nivel alto de resiliencia. Bajo esta misma lógica, se pudo llegar a la misma conclusión para los cinco factores que conforman la escala.

Para el total de la escala, así como para sus factores, los padres fueron quienes mostraron los niveles más altos de resiliencia, seguidos por las tutoras y finalmente las madres de familia.

Tabla 2

Puntaje en la escala total y por factores de la resiliencia.

\begin{tabular}{lcccc}
\hline RESI-M & $\begin{array}{c}\text { Max. } \\
\text { Puntaje } \\
\text { posible }\end{array}$ & Mín. & Máx. & M \\
\hline $\begin{array}{l}\text { Escala total } \\
\text { Fortaleza y confianza }\end{array}$ & 76 & 75 & 168 & 141.43 \\
en sí mismo & 32 & 12 & 32 & 24.84 \\
Competencia social & 24 & 10 & 24 & 20.55 \\
Apoyo familiar & 20 & 11 & 20 & 17.47 \\
Apoyo social & 20 & 5 & 20 & 15.80 \\
Estructura & & & & \\
\hline
\end{tabular}

Correlación entre las variables componentes del modelo

Las variables componentes del modelo para explicar la sintomatología del TEPT en los cuidadores incluyeron: el tiempo transcurrido desde la quemadura, la edad del paciente al momento de la quemadura, el TBSA quemado del paciente, la resiliencia y los tres grupos sintomáticos del TEPT (reexperimentación, evitación y activación) de los cuidadores. Los resultados de estas correlaciones se presentan en la Tabla 3, donde la severidad de los tres grupos sintomáticos únicamente se asoció con la resiliencia de los cuidadores. 
Tabla 3

Correlación entre las variables componentes del modelo

\begin{tabular}{|c|c|c|c|c|c|}
\hline & $\begin{array}{l}\text { Tiempo desde la } \\
\text { quemadura }\end{array}$ & $\begin{array}{c}\text { Edad del paciente momento } \\
\text { de la quemadura }\end{array}$ & TBSA & $\begin{array}{l}\text { Resiliencia de los } \\
\text { cuidadores }\end{array}$ & Reexperimentación \\
\hline $\begin{array}{l}\text { Edad del paciente } \\
\text { momento de la } \\
\text { quemadura }\end{array}$ & $-.751 * *$ & & & & \\
\hline TBSA & .180 & -.031 & & & \\
\hline $\begin{array}{l}\text { Resiliencia de los } \\
\text { cuidadores }\end{array}$ & .100 & .047 & .069 & & \\
\hline Reexperimentación & -.165 & .070 & .021 & $-.360 * *$ & \\
\hline Evitación & -.136 & .000 & .088 & $-.515 * *$ & $.753 * *$ \\
\hline Activación & -.141 & .151 & .011 & $-.443 * *$ & $.648 * *$ \\
\hline
\end{tabular}

**. La correlación es significativa al nivel .01 (bilateral).

\section{Modelamiento de Ecuaciones Estructurales}

Desde la perspectiva teórica del TEPT (Ehlers \& Clark, 2000), se señala un vínculo más fuerte entre los síntomas de evitación y experimentación (que con respecto a los de activación). Dicha perspectiva propone que la evitación cognitiva interfiere con la formación de una memoria del trauma más completa, lo que impide a las personas controlar los pensamientos intrusivos.

Adicionalmente, dado que los síntomas del TEPT no se relacionaron con ninguna otra variable más que con la resiliencia de los cuidadores, se consideró importante indagar, específicamente, en cuáles aspectos de la resiliencia de los cuidadores se encontraban relacionados con su sintomatología.

Se realizó el modelo hipotético de la Figura 1, donde se incluyeron como variables a predecir los síntomas de evitación y experimentación, y los cinco factores que componen la RESI-M como variables predictoras. Se buscó confirmar el primer modelo hipotético (Figura 1), en donde se identificaron los parámetros no significativos, los cuales se eliminaron uno a uno dando lugar a nuevas estimaciones.

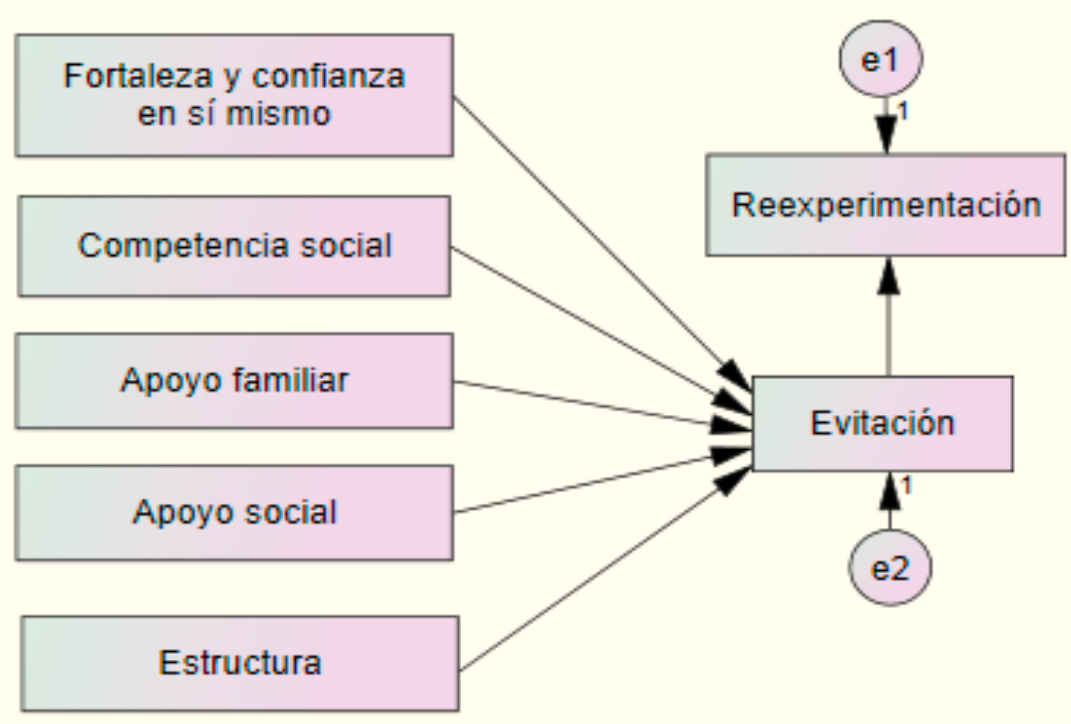

Figura 1. Modelo hipotético para explicar los síntomas del TEPT en los cuidadores. 
Se estimaron un total de cinco modelos, donde los parámetros de los factores de Estructura, Apoyo familiar, Competencia social y Apoyo social, fueron eliminados respectivamente del modelo. En la Figura 2 se representa el modelo final, en el cual se presenta como único predictor de los síntomas del TEPT, el factor de Fortaleza y confianza en sí mismo. Este factor obtuvo un peso de regresión de -.657 $(\mathrm{p}=.001)$ hacia los síntomas de evitación, donde a su vez estos tuvieron un efecto de $.712(\mathrm{p}=.001)$ sobre los síntomas de reexperimentación. El efecto indirecto de la Fortaleza y confianza en sí mismo sobre los síntomas de reexperimentación en los cuidadores fue de -.468. De acuerdo a los límites propuestos para los índices de bondad de ajuste, el modelo final resultó ser adecuado $\left(\mathrm{X}^{2}=.416 ; \mathrm{p}=.519 ; \mathrm{X}^{2} / \mathrm{gl}=.416 ; \mathrm{GFI}=.995\right.$; $\mathrm{AGFI}=.967 ; \mathrm{NFI}=.993 ; \mathrm{TLI}=1.029 ; \mathrm{CFI}=1.000$; RMSEA $=.000)$. El factor de Fortaleza y la confianza en sí mismo de la RESI-M explicó el 43\% de la sintomatología de evitación, y en conjunto, ambas variables explicaron el $51 \%$ de la varianza de los síntomas de reexperimentación.

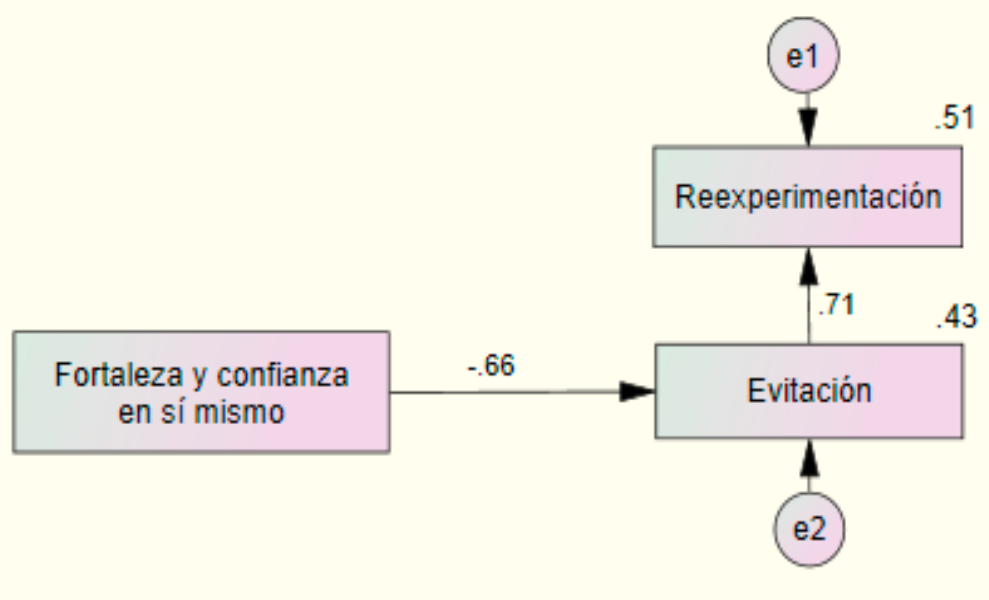

Figura 2. Modelo final para explicar los síntomas del TEPT en los cuidadores.

\section{Discusión y conclusiones}

Altos niveles de resiliencia fueron encontrados en los cuidadores de los pacientes, hallazgo que difiere de estudios previos realizados con padres y madres de niños con quemaduras (McGarry et al., 2013). No obstante, nuestros resultados son semejantes a los encontrados por otros estudios en población adulta mexicana (González-Arratia, 2011; Miaja \& Moral, 2014).

La severidad de la sintomatología del TEPT en los cuidadores resultó ser variable. Los síntomas del TEPT pueden afectar al bienestar y la calidad de vida de las personas, haciéndoles difícil continuar con sus actividades diarias. Incluso si la persona no cumple con los criterios para el trastorno, resulta importante abordar estos síntomas. El modelo de este estudio propone que la resiliencia permite a los cuidadores tener una menor evitación cognitiva del evento, lo que resulta en una memoria del trauma más estructurada y completa, la cual modera la severidad y aparición de los síntomas de reexperimentación (Wenzlaff \& Wegner, 2000).

La resiliencia y los síntomas post-trauma no son fenómenos necesariamente opuestos. Aunque los síntomas del TEPT estuvieron presentes en los cuidadores, la muestra en conjunto obtuvo altos puntajes de resiliencia, lo que contribuye a apoyar la noción de la resiliencia como un amortiguador de consecuencias psicológicas negativas (Olsson et al., 2003).

A diferencia de otros resultados, la edad de los pacientes al momento de la quemadura no se relacionó con las variables de los cuidadores (Odar et al., 2013). De manera similar, el TBSA quemado no tuvo influencia sobre la sintomatología del TEPT en los cuidadores, resultado que difiere al reportado por Rizzone et al. (1994), en donde el TBSA fue encontrado como predictor de dichos síntomas. Bakker et al. (2010) proponen que los síntomas del TEPT en padres y madres de niños con quemaduras disminuyen con el paso del tiempo. En nuestro estudio, el tiempo transcurrido desde la lesión 
no tuvo una influencia significativa sobre la severidad de los síntomas en los cuidadores.

Este estudio cuenta con varias limitaciones. Una de las limitaciones más fuertes consistió en el tamaño de la muestra. Para futuros estudios, se recomienda la posibilidad de incluir pacientes y cuidadores de otros hospitales y centros médicos pertenecientes a diferentes regiones de México. Otra gran limitante consistió en el tiempo dedicado a evaluar a los participantes, debido a que fue planeado que las evaluaciones del estudio no interfirieran con las actividades médicas realizadas. En consecuencia, el número de variables de estudio se vio limitado. Al no evaluar otros trastornos del estado de ánimo y ansiedad en los cuidadores, se desconoce la existencia de una posible comorbilidad. Adicionalmente, se hace la recomendación de contemplar la posibilidad de realizar entrevistas a los participantes del estudio, con el propósito de robustecer la información.

El sesgo en las respuestas en relación al lugar de la evaluación no puede ser descartado. Una recomendación para futuros estudios consiste en conducir la recolección de la información en ambientes menos asociados a la historia traumática de los participantes, donde este tipo de escenario pueda reducir las influencias del ambiente. No obstante, resulta casi inevitable poder conducir estas evaluaciones fuera de un recinto médico, por lo que crear un ambiente ameno para los participantes puede contribuir a reducir los sesgos asociados con este tipo de locación.

Puesto que la mayor parte de la muestra de cuidadores se encontró constituida por madres, es recomendable para futuros estudios sobre el área de interés, así como estudios que contemplen aspectos relacionados con niños y adolescentes, incluir un número de padres similar al de las madres. Lo anterior, con la finalidad de poder obtener mayor información sobre el fenómeno objeto de estudio de acuerdo al sexo de los cuidadores.

Finalmente, de acuerdo a los resultados puede concluirse que a mayor fortaleza y confianza en los cuidadores, menor severidad en sus síntomas de evitación, lo que a su vez resulta en una menor reexperimentación relacionada con el accidente de la quemadura. Es importante mencionar que las reacciones post-quemadura de los cuidadores pueden repercutir en el bienestar de sus hijos, por lo que un mejor entendimiento de los factores relacionados con la adaptación de estos cuidadores tendrá como resultado una mejor atención por parte de los servicios de salud.

\section{Referencias}

American Psychiatric Association (2000). Diagnostic and statistical manual of mental disorders: DSM-IV-TR. Washington, DC: American Psychiatric Association.

Bakker, A., Maertens, K., Van Son, M. \& Van Loey, N. (2013). Psychological consequences of pediatric burns from a child and family perspective: a review of the empirical literature. Clinical Psychology Review, 33(3), 361-371. doi:10.1016/j.cpr.2012.12.006

Bakker, A., Van Loey, N., Van der Heijden, P. \& Van Son, M. (2012). Acute stress reactions in couples after a burn event to their young child. Journal of Pediatric Psychology, 37(10), 1127-1135. doi:10.1093/jpepsy/jss083

Bakker, A., Van Loey, N., Van Son, M. \& Van der Heijden, P. (2010) Brief report: mothers' long-term posttraumatic stress symptoms following a burn event of their child. Journal of Pediatric Psychology, 35(6), 656-661. doi:10.1093/jpepsy/jsp090

Bobes, J., Calcedo-Barba, A., García, M., François, M., Rico-Villademoros, E., González, M., Bascarán, M., Bousoño, M. \& Grupo español de trabajo para el estudio del trastorno por estrés postraumático (2000). Evaluación de las propiedades psicométricas de la versión española de cinco cuestionarios para la evaluación del trastorno de estrés postraumático. Actas Españolas de Psiquiatría, 28(4), 207-218. Recuperado de http://www.unioviedo.net/psiquiatria/publicaciones/documentos/2000/2000_Bobes_Evaluacion.pdf

Breton, J., Valla, J. \& Lambert, J. (1993). Industrial disaster and mental health of children and their parents. Journal of the American Academy of Child and Adolescent Psychiatry, 32(2), 438-445. doi:10.1097/00004583-199303000-00028

Davidson, J., Book, S., Colket, J., Tupler, L., Roth, S., David, D., Hertzberg, M., Mellman, T., Beckham, J., Smith, R., Davison, R., Katz, R. \& Feldman, M. (1997). Assessment of a new self-rating scale for post-traumatic stress disorder. Psychological Medicine, 27(1), 153-160. doi:10.1017/S0033291796004229

Dirección General de Epidemiología (2012). Información Epidemiológica de Morbilidad, Anuario 2011. Versión Ejecutiva. México: Secretaría de Salud. ISBN: en trámite.

Ehlers, A. \& Clark, D. (2000). A cognitive model of posttraumatic stress disorder. Behaviour Research and Therapy, 38, 319-345. doi:10.1016/S0005-7967(99)00123-0

El Hamaoui, Y., Yaalaoui, S., Chihabeddine, K., Boukind, E. \& Moussaoui, D. (2006). Depression in mothers of burned children. Archives of Women's Mental Health, 9(3), 117-119. doi:10.1007/ s00737-006-0124-1

González-Arratia, N. I. (2011). Resiliencia en madres e hijos con cáncer. Psicooncología, 8(1), 113-123. doi:10.5209/rev_PSIC.2011. v8.n1.9

Hooper, D., Coughlan, J. \& Mullen, M. (2008). Structural equation modelling: Guidelines for determining model fit. Electronic Journal of Business Research Methods, 6(1), 53-60. Recuperado de http://arrow.dit.ie/cgi/viewcontent.cgi?article=1001\&contex $\mathrm{t}=$ buschmanart

Kazak, A., Kassam-Adams, N., Schneider, S., Zelikovsky, N., Alderfer, M. \& Rourke, M. (2006). An integrative model of pediatric medical traumatic stress. Journal of Pediatric Psychology, 31(4), 343-345. doi:10.1093/jpepsy/jsj054

Langeland, W. \& Olff, M. (2008). Psychobiology of posttraumatic stress disorder in pediatric injury patients: a review of the literature. Neuroscience and Biobehavioral Review, 32(1e), 161-174. doi:10.1016/j.neubiorev.2007.07.002

McGarry, S., Girdler, S., McDonald, A., Valentine, J., Wood, F. \& Elliott, C. (2013). Paediatric medical trauma: The impact on parents of burn survivors. Burns, 39(6), 1114-1121. doi:10.1016/j. burns.2013.01.009 
Miaja, M. \& Moral, J. (2014). Predicción de las respuestas psicológicas del duelo ante la pérdida de salud por variables de personalidad, religiosidad sociodemográficas, y existenciales. Revista Salud y Sociedad, 5(3), 254-272. Recuperado de www.saludysociedad.cl/ index.php/main/article/view/190

Odar, C., Kirschman, K., Pelley, T., Butz, C., Besner, G. \& Fabia, R. (2013). Prevalence and correlates of posttraumatic stress in parents of young children postburn. Journal of Burn Care \& Research, 34(3), 299-306. doi: 10.1097/BCR.0b013e31825ae15d

Olsson, C., Bond, L., Burns, J., Vella-Brodrick, D. \& Sawyer, S. (2003). Adolescent resilience: a concept analysis. Journal of Adolescence, 26, 1-11. doi:10.1016/S0140-1971(02)00118-5

Palomar, J. \& Gómez Valdez, N. (2010). Desarrollo de una escala de medición de la resiliencia con mexicanos (RESI-M). Interdisciplinaria, 27(1), 7-22. Recuperado de http://www.scielo.org.ar/ pdf/interd/v27n1/v27n1a02.pdf
Phillips, C. \& Rumsey, N. (2008). Considerations of the provision of psychosocial services for families following paediatric burn injury-a quantitative study. Burns, 34(1), 56-62. doi:10.1016/j. burns.2006.12.003

Rizzone, L., Stoddard, F., Murphy, J. \& Kruger, L. (1994). Posttraumatic stress disorder in mothers of children and adolescents with burns. Journal of Burn Care \& Rehabilitation, 15(2), 158-163. doi:10.1097/00004630-199403000-00010

Saxe, G., Stoddard, F., Hall, E., Chawla, N., Lopez, C., Sheridan, R., ... \& Yehuda, R. (2005). Pathways to PTSD, part I: Children with burns. American Journal of Psychiatry, 162(7), 1299-1304. doi:10.1176/appi.ajp.162.7.1299

Wenzlaff, R. \& Wegner, D. (2000). Thought suppression. Annual Review of Psychology, 51, 59-91. doi:10.1146/annurev. psych.51.1.59 